Department of Medicine, Seoul, Korea, Republic of (South Korea); ${ }^{2}$ Samsung Medical Center, Sungkyunkwan University School of Medicine, Department of Medical Humanities, Seoul, Korea, Republic of (South Korea)

Background: Previous studies have shown that cardiovascular risk is increased in patients with gout. There are many studies on the effect of uric acid lowering therapy on CV risk in gout patients, but few studies have compared allopurinol and benzbromarone.

Objectives: A nationwide population-based cohort study is designed to compare cardiovascular risk according to the treatment of allopurinol and benzbromarone in Korean gout patients.

Methods: We used South Korea's database of the Health Insurance Review and Assessment service (HIRA) to identify gout patients 18 years of age or older who newly started allopurinol or benzbromarone between 2009 and 2015. The start date of allopurinol or benzbromarone is defined as the index date. We excluded patients who have been prescribed uric acid lowering agents or have been on dialysis for one year prior to the index date. During the study period, patients who used uric acid lowering agents other than allopurinol and benzbromarone or who used both drugs in combination were also excluded from the study. The primary outcome of the study was the occurrence of a composite cardiovascular endpoint, which included coronary revascularization, hospitalization due to MI, ischemic stroke, and transient ischemic attack (TIA). Cox proportional hazard regression analysis and Kaplan-Meier curves were used for the analysis.

Results: 257,097 allopurinol initiators and 7,868 benzbromarone initiators were included in the study. The mean age was 54.4 years, $86 \%$ were male. The mean adherence of drug administration was $68.2 \%$ for allopurinol initiators and $75.5 \%$ for benzbromarone initiators. In baseline, the benzbromarone initiator had more cardiovascular comorbidities and related drug administration than the allopurinol initiator. In allopurinol and benzbromarone initiators, the adjusted hazard ratio (aHR) of the composite CV endpoint was 1.01 (95\% Cl 0.83-1.21), which was not significantly different. No significant difference was found between the two groups in each of the items of the composite CV endpoint and hospitalization for heart failure. The results did not change even when 1:3 propensity score matching was performed for baseline characteristics. In subgroup analysis of high risk patients with cardiovascular disease, there was no significant difference between allopurinol and benzbromarone initiators. However, when the analysis was limited to the group taking allopurinol $\geq 200 \mathrm{mg}$ and benzbromarone $\geq 50 \mathrm{mg}$, there was no difference in primary outcome and other outcomes, but the risk of coronary revascularization was higher in benzbromarone initiator (aHR 1.58, $95 \%$ Cl 1.16-2.14)

Conclusion: In our study, there was no significant difference in cardiovascular risk between allopurinol initiator and benzbromarone initiator. In the high risk group of cardiovascular disease, there was no difference in risk between the two drugs.

Disclosure of Interests: None declared

DOI: 10.1136/annrheumdis-2021-eular.3952

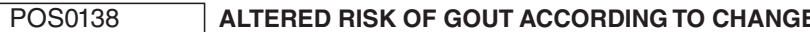 OF METABOLIC PARAMETERS IN YOUNG ADULTS}

Y. Eun ${ }^{1}$, I. Y. Kim², K. D. $\mathrm{Han}^{3}$, S. Y. Kang ${ }^{1}$, S. Lee ${ }^{1}$, H. S. Cha ${ }^{1}$, E. M. Koh¹, H. Kim ${ }^{4}$, J. Lee'. ' Samsung Medical Center, Sungkyunkwan University School of Medicine, Department of Medicine, Seoul, Korea, Rep. of (South Korea); ${ }^{2}$ National Police Hospital, Department of Medicine, Seoul, Korea, Rep. of (South Korea); ${ }^{3}$ Soongsil University, Department of Statistics and Actuarial Science, Seoul, Korea, Rep. of (South Korea); ${ }^{4}$ Samsung Medical Center, Sungkyunkwan University School of Medicine, Department of Medical Humanities, Seoul, Korea, Rep. of (South Korea)

Background: Many studies have shown a link between gout and metabolic syndrome (MetS). It is well known that lifestyle modifications such as weight reduction and abstinence from alcohol are effective in the treatment of gout, but data are lacking on how exactly the change of metabolic parameters affects gout. Objectives: The purpose of this study was to investigate the relationship between gout risk and metabolic parameters in a nationwide population based young adult cohort, and to determine whether changes in metabolic parameters affect gout risk changes.

Methods: Among adults aged 20-39 years who participated in national health check-up programs from 2009 to 2012 , a total of $6,290,914$ subjects were included in the study, excluding subjects who were previously diagnosed with gout. To determine the effect of changes in metabolic parameters on gout incidence, 2,701,138 subjects who participated in the health examination once more 2 years later were used for the analysis. Outcome was defined as the occurrence of gout, when the ICD-10 code (M10) was registered twice in the claim database. The Cox proportional hazard model and Kaplan Meier curve were used for the analysis.

Results: The incidence rate of gout was higher in those with MetS compared to those without (10.1 vs. 3.6 per 1,000 person-years). The risk of gout in people with MetS was 85\% higher (adjusted HR 1.85, 95\% Cl 1.83-1.87) and was more significant in men than in women (adjusted HR 1.88 in male and 1.56 in female). Each component of MetS was also associated with increased gout risk, and hypertriglyceridemia showed the highest adjusted HR. The greater the number of MetS components, the higher the gout risk. The risk of gout was $70 \%$ higher in those who had MetS consistently (adjusted HR 1.71, 95\% Cl 1.67-1.75) and 44\% higher in those with newly developed MetS (adjusted HR 1.47, 95\% CI 1.40-1.48) than those who did not have MetS at the two health examinations. Similar risk patterns were observed according to the change of each metabolic parameter Among the metabolic parameters, the change in hypertriglyceridemia was associated with the greatest difference in the change in gout risk.

Conclusion: In young adults, MetS was associated with a higher risk of gout, especially with more components, the higher the risk. Since the occurrence of MetS is associated with an increased risk of gout, prevention of MetS would be important to reduce gout incidence.

Disclosure of Interests: None declared

DOI: 10.1136/annrheumdis-2021-eular.4032

\section{POS0139 TWO-YEAR REDUCTION OF URATE LOAD IN DUAL-ENERGY CT DURING A TREAT-TO-TARGET APPROACH IN GOUT PATIENTS: RESULTS FROM A LONGITUDINAL STUDY (NOR-GOUT)}

T. Uhlig ${ }^{1}$, T. Eskild ${ }^{2}$, L. F. Karoliussen ${ }^{1}$, T. K. Kvien ${ }^{1}$, E. A. Haavardsholm ${ }^{1}$, N. Dalbeth ${ }^{3}$, H. B. Hammer ${ }^{1}{ }^{1}$ Diakonhjemmet Hospital, Rheumatology, Oslo, Norway; ${ }^{2}$ Diakonhjemmet Hospital, Clinical Service Radiology, Oslo, Norway; ${ }^{3}$ University of Auckland, Medicine, Auckland, New Zealand

Background: Dual-energy computed tomography (DECT) detects urate depositions, and is included in the ACR/EULAR classification criteria for gout. There is lack of longitudinal studies in large patient populations for changes in urate deposition measured by DECT during urate lowering therapy (ULT).

Objectives: To explore by DECT the longitudinal changes urate depositions during a treat-to-target approach in gout with ULT.

Methods: In a prospective observational study, patients with crystal-proven gout were included if they presented after a recent gout flare and with increased serum urate levels $(>360 \mu \mathrm{mol} / \mathrm{L} />6 \mathrm{mg} / \mathrm{dl})$. In a treat-to-target approach they received ULT with escalating drug doses with monthly follow-up during the firs year until the treatment target was met with serum urate $<360 \mu \mathrm{mol} / \mathrm{L}$ or 360 $\mu \mathrm{mol} / \mathrm{L}$ if tophi.

A DECT scanner (General Electric Discovery CT750 HD) acquired data from bilateral forefeet and ankles at $80 \mathrm{KW}$ and $140 \mathrm{KV}$, processed with a software with a 2-material decomposition algorithm which colour codes urate. Follow-up DECT was performed after one and two years.

Images were scored with a semiquantitative method (Bayat et al. 2015) by one experienced radiologist in known order and blinded to serum urate and clinical data. Each scan assessed four regions: the first metatarsophalangeal (MTP1) joint, the other joints of the toes, the ankles and midfeet, and all visible tendons in the feet and ankles. Each region was then scored according to the maximum amount of urate deposition observed on visual inspection ( $0=$ no deposits, $1=$ dots, $2=$ single deposit, $3=$ more than one deposit). $A$ total DECT sum score was derived by adding all values from the four regions, with a maximum score of 12

Results: DECT was at baseline measured in 187 of 211 patients. $95.2 \%$ were males, mean (SD) age was 56.7 (13.7) years, disease duration 8.1 (7.9) years) and $17 \%$ had clinical tophi.

The total DECT scores and all subscores decreased from baseline to 2 years ( $p<0.001$ for all comparisons versus baseline), table 1 .

The mean (SD) serum urate level ( $\mu \mathrm{mol} / \mathrm{L})$ decreased from $501(80)$ at baseline to 311 (48) at 12 months and 322 (67) at 24 months. Reaching the treatment target $<360 \mu \mathrm{mol} / \mathrm{L}$ after year 1 or 2 was only numerically but not statistically related to reductions in DECT scores. Patients with clinical tophi at baseline had larger reductions in total DECT scores after $1(3.4$ vs. $1.5, p<0.01)$ and 2 years $(6.5$ vs. 2.3, $p<0.001$ ) than patients without tophi.

Conclusion: During a treat-to-target approach urate deposition visualised by DECT were clearly reduced in ankles and feet after 1 year with further reduction after 2 years.

Table 1.

\begin{tabular}{lccc}
\hline & $\begin{array}{c}\text { Baseline }(n=187) \\
\text { Mean }(\mathrm{SD})\end{array}$ & $\begin{array}{c}1 \text { year }(\mathrm{n}=157) \\
\text { Mean }(\mathrm{SD})\end{array}$ & $\begin{array}{c}2 \text { years }(\mathrm{n}=166) \\
\text { Mean }(\mathrm{SD})\end{array}$ \\
\hline MTP1 (0-3) & $1.4(2.0)$ & $1.0(1.7)$ & $0.6(1.3)$ \\
Toes (0-3) & $1.0(1.8)$ & $0.6(1.4)$ & $0.3(1.0)$ \\
Ankle/Midfoot $(0-3)$ & $1.2(2.1)$ & $0.7(1.6$ & $0.3(1.0)$ \\
Tendons (0-3) & $1.0(1.7)$ & $0.5(1.2)$ & $0.3(0.8)$ \\
Sum score (0-12) & $4.6(6.4)$ & $2.8(4.7)$ & $1.5(3.2)$ \\
\hline
\end{tabular}


Disclosure of Interests: Till Uhlig Speakers bureau: Grünenthal, Novartis, Consultant of: Grünenthal, Novartis, Tron Eskild: None declared, Lars Fridtjof Karoliussen: None declared, Tore K. Kvien Consultant of: AbbVie, MSD, UCB, Hospira/Pfizer, Eli-Lilly, Roche, Hikma, Orion, Sanofi, Celltrion, Sandoz, Biogen, Amgen, Egis, Ewopharma, Mylan, Grant/research support from: BMS, AbbVie, MSD, UCB, Hospira/Pfizer, Eli-Lilly, Espen A Haavardsholm Consultant of: Pfizer, UCB, Eli Lilly, Celgene, Janssen-Cilag, AbbVie and Gilead, Nicola Dalbeth Speakers bureau: Menarini, AstraZeneca, Takeda, S. Nicolaou, Consultant of: AstraZeneca, Fonterra, Takeda, Pfizer, Cymabay, Crealta, Grant/research support from: AstraZeneca, Siemens Healthcare, Hilde Berner Hammer Consultant of: AbbVie, Lilly and Novartis

DOI: 10.1136/annrheumdis-2021-eular.751

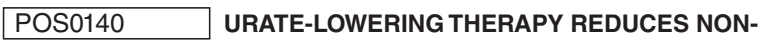 EPISODIC FOOT PAIN IN PATIENTS WHO FAIL TO MEET ACR/EULAR 2015 GOUT CLASSIFICATION CRITERIA: AN EFFECT PREDICTED BY ULTRASOUND}

R. Flood ${ }^{1}$, C. Kirby ${ }^{2}$, Y. Alammari ${ }^{2}$, D. Kane ${ }^{2}$, R. Mullan ${ }^{2}{ }^{1}$ Tallaght University Hospital, Rheumatology, Dublin, Ireland; ${ }^{1}$ Tallaght University Hospital, Rheumatology, Dublin, Ireland

Background: Emerging evidence that the joints of asymptomatic hyperuricaemic individuals contain monosodium urate (MSU) deposits and that alternative presentations of foot pain occur in hyperuricaemia suggests that preclinical phases may occur prior to a first episodic gout attack. (1) This case-control study evaluates urate deposition in hyperuricaemic individuals not fulfilling the current gout classification criteria, as well as a potential therapeutic role for urate lowering therapy (ULT).

Objectives: To investigate whether ULT reduces non-episodic foot pain in patients who fail to meet ACR/EULAR 2015 gout classification criteria.

Methods: Following informed consent, hyperuricaemic individuals with persistent, non-episodic foot pain $(n=53)$ not fulfilling ACR/EULAR 2015 gout classification criteria, were compared with asymptomatic hyperuricaemic controls $(n=18)$. Ultrasound (US) of bilateral first metatarsophalangeal (MTP) joints and features of MSU deposition including double contour (DC) sign, tophus and juxta-articular erosion were recorded. Cases only were treated with febuxostat or allopurinol daily for 6 months. Serum urate, 24-hour and 7-day visual analogue score (VAS) 0-100 mm pain scales and the Manchester Foot Pain and Disability Index (MFPDI) were recorded before treatment and after 3 and 6 months. MTP Ultrasound was repeated after a minimum of 6 months on treatment.

Results: 53 hyperuricaemic individuals with persistent, non-episodic foot pain not meeting the ACR/EULAR 2015 gout classification criteria were recruited. At baseline MTP US DC sign, erosion and tophus occurred in $62.5 \%, 20.8 \%$ and $49 \%$ of cases, respectively. No US features of gout occurred in controls. No significant difference was seen in baseline serum urate between cases $(481 \pm 14 \mathrm{mg} / \mathrm{dL})$ versus controls ( $437 \pm 14 ; p=N S)$. Serum urate in cases fell at 3 months $(325 \pm 25 ; p<0.01)$ and 6 months $(248 \pm 19 ; p<0.01)$. For cases, baseline 24 -hour pain VAS $(46 \pm 3.9)$ reduced at 3 months $(32 \pm 4.1 ; p<0.05)$ and 6 months $(21 \pm 5.2 ; p<0.05)$ of ULT. The 7 -day pain VAS $(59 \pm 3.9)$ decreased at 3 months $(35 \pm 4.5 ; p<0.05)$ and 6 months $(30 \pm 5.3 ; \mathrm{P}<0.05)$. MFPDI $(17 \pm 1.4)$ decreased at 3 month $(13 \pm 1.8 ; \mathrm{p}=<0.05)$ and 6 months $(11 \pm 2.2 ; p=<0.05)$. When cases were grouped according to the presence $(\mathrm{N}=33)$ or absence $(\mathrm{N}=18)$ of $\mathrm{DC}$ sign on baseline US, no differences were observed for baseline pain scores. Following ULT however, 24-hour pain VAS were significantly lower in DC positive patients at 3 months $(22 \pm 4.48$ DC positive vs 42 \pm 6.14 DC negative; $p<0.05)$ and 6 months $(12 . \pm 5.4$ vs $33 \pm 8.4 ; p<0.05)$. The 7 -day pain VAS were significantly lower in DC positive patients at 3 months $(23 \pm 4.6$ vs $47 \pm 6.6 ; p<0.05)$ and MFDPI were significantly lower in DC positive patients at 3 months $(10 \pm 1.9$ DC positive vs $19 \pm 2.9 \mathrm{DC}$ negative; $p<0.05)$.

Conclusion: These findings indicate that persistent, non-episodic foot pain in hyperuricaemia is both associated with US features of MSU deposition and is responsive to ULT. Symptomatic hyperuricaemia occurring prior to episodic gout therefore represents an earlier or alternative disease presentation. Changes to the ACR/ EULAR classification criteria to include non-episodic foot pain in the presence of US features of gout may increase the sensitivity of disease classification at an early stage, leading to improved future treatment strategies and long-term outcomes.

\section{REFERENCES:}

[1] Stewart S, Dalbeth N, Vandal AC, Rome K. Characteristics of the first metatarsophalangeal joint in gout and asymptomatic hyperuricaemia: $A$ cross-sectional observational study. J Foot Ankle Res. 2015;8(1):1-8.

Disclosure of Interests: None declared

DOI: 10.1136/annrheumdis-2021-eular.1571

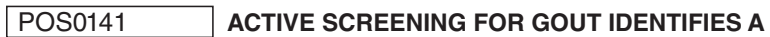 LARGER CARDIOVASCULAR POPULATION AT HIGH MORTALITY RISK}

S. Ruiz-Simón ${ }^{1}$, I. Calabuig ${ }^{2}$, M. Gomez-Garberi ${ }^{3}$, M. Andrés ${ }^{1,2}$. ${ }^{1}$ Miguel Hernandez University, Clinical Medicine, San Juan de Alicante, Spain; ${ }^{2}$ Alicante General University Hospital-ISABIAL, Rheumatology, Alicante, Spain; ${ }^{3}$ San Juan de Alicante University Hospital, Urology, San Juan de Alicante, Spain

Background: We have recently revealed by active screening that about a third of gout cases in the cardiovascular population is not registered in records [1], highlighting the value of field studies.

Objectives: To assess whether gout screening in patients hospitalized for cardiovascular events may also help identify patients at higher risk of mortality after discharge.

Methods: A retrospective cohort field study, carried out in 266 patients admitted for cardiovascular events in the Cardiology, Neurology and Vascular Surgery units of a tertiary centre in Spain. The presence of gout was established by records review and face-toface interview, according to the 2015 ACR/EULAR criteria. The occurrence of mortality during follow-up and its causes were obtained from electronic medical records. The association between gout and subsequent mortality was tested using Cox regression models. Whether covariates affect the gout-associated mortality was also studied.

Results: Of 266 patients recruited at baseline, 17 were excluded due to loss to follow-up (>6mo), leaving a final sample of 249 patients $(93.6 \%)$. Thirty-six cases (14.5\% of the sample) were classified as having gout: twenty-three $(63.9 \%)$ had a previously registered diagnosis, while $13(36.1 \%)$ had not and was established by the interview.

After discharge, the mean follow-up was 19.9 months $(S D \pm 8.6)$, with a mortality incidence of 21.6 deaths per 100 patient-years, $34.2 \%$ by cardiovascular causes. Gout significantly increased the risk of subsequent all-cause mortality, with a hazard ratio (HR) of $2.01(95 \% \mathrm{Cl} 1.13$ to 3.58$)$. When the analysis was restricted to gout patients with registered diagnosis, the association remained significant (HR 2.89; 95\% Cl 1.54 to 5.41 ).

The adjusted HR for all-cause mortality associated with gout was $1.86(95 \% \mathrm{C}$ 1.01-3.40). Regarding the causes of death, both cardiovascular and non-cardiovascular were numerically increased.

Secondary variables rising the mortality risk in those with gout were age (HR $1.07 ; 1.01$ to 1.13 ) and coexistent renal disease (HR 4.70; 1.31 to 16.84 ), while gender, gout characteristics and traditional risk factors showed no impact.

Conclusion: Gout was confirmed an independent predictor of subsequent allcause mortality in patients admitted for cardiovascular events. Active screening for gout allowed identifying a larger population at high mortality risk, which may help tailor optimal management to minimize the cardiovascular impact. REFERENCES:

[1] Calabuig I, et al. Front Med (Lausanne). 2020 Sep 29;7:560.

Disclosure of Interests: Silvia Ruiz-Simón: None declared, Irene Calabuig: None declared, Miguel Gomez-Garberi: None declared, Mariano Andrés Speakers bureau: Grunenthal, Menarini, Consultant of: Grunenthal, Grant/research support from: Grunenthal

DOI: 10.1136/annrheumdis-2021-eular.1872

\section{Fine-tuning strategies (beyond treatments) to reduce the impact of PsA}

\section{POS0142 MINIMAL DISEASE ACTIVITY IN PATIENTS WITH PSORIATIC ARTHRITIS AND ASSOCIATED FACTORS:} REAL LIFE DATA FROM A SINGLE CENTER

M. Bektaș ${ }^{1}$, B. Çelik ${ }^{2}$, B. Ince ${ }^{1}$, Y. Yalçınkaya ${ }^{1}$, B. Artim-Esen ${ }^{1}$, A. Gül ${ }^{1}$, M. L. Ocal' ${ }^{1}$, M. Inanc ${ }^{1}{ }^{1}$ Istanbul Faculty of Medicine, Internal Medicine, Rheumatology Division, Istanbul, Turkey; ${ }^{2}$ Istanbul Faculty of Medicine, Istanbul Faculty of Medicine, Istanbul, Turkey

Background: Psoriatic arthritis (PsA) is a heterogeneous disease and GRAPPA have proposed Minimal disease activity (MDA) as a composite outcome measure and has been validated in PsA.

Objectives: In this study, we aimed to evaluate the characteristics, MDA frequencies, first biological disease modifying antirheumatic drugs (b-DMARD) continuation rate and associated factors in our PsA cohort.

Methods: PsA patients who fulfilled the CASPAR classification criteria and had at least six months of follow-up data were evaluated cross-sectionally for MDA.Clinical data were collected from patient charts with standard forms.b-DMARD treatment was initiated in patients who did not respond to at least one conventional synthetic (cs) 